\title{
Tree-level new physics searches in semileptonic $B$ decays at Belle
}

\section{Christoph Schwanda*}

Institute of High Energy Physics, Austrian Academy of Sciences, Austria

E-mail: Christoph. Schwanda@oeaw.ac.at

Semileptonic $B$ decays involving a $\tau$ lepton can be mediated by a charged Higgs boson in New Physics scenarios including an extended Higgs sector, turning these decays into sensitive probes for physics beyond the Standard Model. Indeed, current measurements of the decays $B \rightarrow D^{(*)} \tau \nu$ show an excess of the observed branching fractions with respect to the Standard Model expectations. In this article we review two recent experimental studies of semitauonic $B$ decays based on the full data sample of the Belle experiment, a measurement of $B \rightarrow D^{*} \tau \nu$ using the semileptonic tagging method and a search for the decay $B \rightarrow \pi \tau \nu$.

XIII International Conference on Heavy Quarks and Leptons

22-27 May, 2016

Blacksburg, Virginia, USA

${ }^{*}$ Speaker. 


\section{Introduction}

In the Standard Model (SM) semileptonic $B$ meson decays are mediated by the $W$ boson. In New Physics (NP) scenarios involving an extended Higgs sector, these decays can also occur by the exchange of a charged Higgs boson, in particular if the mass of the charged lepton is large [1, $2,3,4]$. This turns semileptonic $B$ meson decays involving a $\tau$ lepton into a sensitive probe of physics beyond the SM. In particular, the current interest in semitauonic decays was sparked by the measurement of an excess of $B \rightarrow D^{(*)} \tau \nu$ decays by the BaBar experiment [5]. This observation has been confirmed by the Belle [6] and LHCb experiments [7].

In this article, we review a new Belle measurement of the decay $B \rightarrow D^{*} \tau \nu$ using the semileptonic tagging method, which has been submitted for publication to Physical Review D [8]. A combination of this new measurement with the existing data by the Heavy Flavour Averaging Group is presented. Finally, we also review a search for the decay $B \rightarrow \pi \tau \nu$ in the Belle data [9].

\section{The Belle dataset}

The Belle detector is a large-solid-angle magnetic spectrometer, located in the interaction region of the KEKB machine which collides $3.5 \mathrm{GeV}$ positrons and $8 \mathrm{GeV}$ electrons [10] at the center-of-mass (c.m.) energy of the $\Upsilon(4 S)$-resonance $(10.58 \mathrm{GeV})$. Belle consists of a silicon vertex detector, a 50-layer central drift chamber (CDC), an array of aerogel threshold Cherenkov counters (ACC), a barrel-like arrangement of time-of-flight scintillation counters (TOF), and an electromagnetic calorimeter comprised of CsI(Tl) crystals (ECL), located inside a super-conducting solenoid coil that provides a $1.5 \mathrm{~T}$ magnetic field. The iron flux return is instrumented to detect $K_{L}$ mesons and to identify muons (KLM).

Electron candidates are identified using the ratio of the energy detected in the ECL to the track momentum, the ECL shower shape, position matching between track and ECL cluster, the energy loss in the CDC $(d E / d x)$ and the response of the ACC counters. Muons are identified based on their penetration range and transverse scattering in the KLM detector. For both electrons and muons the efficiency of lepton identification is above $90 \%$. Pions are misidentified as electrons and muons with a probability of $\approx 0.1 \%$ and $\approx 1 \%$, respectively.

Belle operated from 1999 to 2010 and accumulated an integrated luminosity of about $711 \mathrm{fb}^{-1}$ on the $\Upsilon(4 S)$-resonance. There, $B \bar{B}$ pairs are produced at threshold with a cross-section of $1.1 \mathrm{nb}$, resulting in a data sample of about 772 million $B \bar{B}$ events.

\section{3. $B \rightarrow D^{*} \tau \nu$ with semileptonic tagging}

The new Belle measurement [8] of the ratio $\mathscr{R}\left(D^{*}\right)$, defined as

$$
\mathscr{R}\left(D^{*}\right)=\frac{\mathscr{B}\left(B \rightarrow D^{*} \tau \nu\right)}{\mathscr{B}\left(B \rightarrow D^{*} \ell v\right)}, \quad \ell=e, \mu
$$

proceeds by reconstructing $D^{*+}$ mesons in hadronic events [11]. Candidate $D^{*+}$ mesons are searched for in the decay modes $D^{*+} \rightarrow D^{0} \pi^{+}$and $D^{*+} \rightarrow D^{+} \pi^{0}$. Neutral $D$ mesons are reconstructed in the following decay modes: $D^{0} \rightarrow K^{-} \pi^{+}, K_{S}^{0} \pi^{0}, K^{+} K^{-}, \pi^{+} \pi^{-}, K_{S}^{0} \pi^{+} \pi^{-}, K^{-} \pi^{+} \pi^{0}$, 
$\pi^{+} \pi^{-} \pi^{0}, K_{S}^{0} K^{+} K^{-}, K^{-} \pi^{+} \pi^{+} \pi^{-}$, and $K_{S}^{0} \pi^{+} \pi^{-} \pi^{0}$. Charged $D$ mesons are searched for in: $D^{+} \rightarrow$ $K_{S}^{0} \pi^{+}, K^{-} \pi^{+} \pi^{+}, K_{S}^{0} \pi^{+} \pi^{0}, K^{+} K^{-} \pi^{+}$, and $K_{S}^{0} \pi^{+} \pi^{+} \pi^{-}$. These modes cover $37 \%$ (22\%) of the $D^{0}$ $\left(D^{+}\right)$decay width.

To tag semileptonic $B$ decays, the reconstructed $D^{*+}$ is combined with a charged lepton candidate (electron or muon) of opposite electric charge and the cosine of the angle between the momentum of the $B$ meson and the $D^{*} \ell$ system in the c.m. frame is calculated, assuming that only the neutrino from the semileptonic decay is not reconstructed,

$$
\cos \theta_{B-D^{*} \ell}=\frac{2 E_{\mathrm{beam}} E_{D^{*} \ell}-m_{B}^{2} c^{4}-M_{D^{*} \ell}^{2} c^{4}}{2\left|\vec{p}_{B}\right| \cdot\left|\vec{p}_{D^{*} \ell}\right| c^{2}},
$$

where $E_{\text {beam }}$ is the c.m. energy of the beam, and $E_{D^{*} \ell}, \vec{p}_{D^{*} \ell}$, and $M_{D^{*} \ell}$ are the energy, momentum, and mass of the $D^{*} \ell$ system, respectively. The $B$ meson mass is designated by $m_{B}$ and $\left|\vec{p}_{B}\right|$ is the magnitude of the $B$ meson 3-momentum, as determined by $\Upsilon(4 S)$ decay kinematics. $D^{*} \ell$ pairs which stem from the decay $B \rightarrow D^{*} \ell v$ (normalization mode) are expected to have a value of $\cos \theta_{B-D^{*} \ell}$ between -1 and +1 . However, pairs which originate from $B \rightarrow D^{*} \tau \nu$ (signal mode) tend to have values of $\cos \theta_{B-D^{*} \ell}$ below the physical region due to the presence of additional particles in the final state.

In the next step, we select events with two tagged $B$ candidates of opposite flavor. The candidate with the lower value of $\cos \theta_{B-D^{*} \ell}$ is considered to be the signal $B$ meson $\left(B_{\text {sig }}\right)$ while the second $B$ candidate is taken as the tag side $\left(B_{\text {tag }}\right)$. For $B_{\text {sig }}$ decaying into $D^{*} \tau \nu$ this assignment is correct in $97 \%$ of the cases. On the tag side we require $-2.0<\cos \theta_{B-D^{*} \ell}^{\operatorname{tag}}<+1.5$. Also, the event must not contain extra charged particles, $K_{S}^{0}$ candidates, or $\pi^{0}$ candidates.

To separate $B \rightarrow D^{*} \tau \nu$ signal and $B \rightarrow D^{*} \ell v$ normalization events, a neural network based on NeuroBayes is trained [12]. The input variables used are: $\cos \theta_{B-D^{*} \ell}^{\text {sig }}$, the missing mass squared $M_{\text {miss }}^{2}=\left(2 E_{\text {beam }}-\sum_{i} E_{i}\right)^{2} / c^{4}-\left|\sum_{i} \vec{p}_{i}\right|^{2} / c^{2}$, and the visible energy $E_{\mathrm{vis}}=\sum_{i} E_{i}$, where $\left(E_{i}, \vec{p}_{i}\right)$ is the 4-momentum of particle $i$ in the c.m. frame. The most powerful input for separating signal and normalization is $\cos \theta_{B-D^{*} \ell}^{\text {sig }}$.

Finally, we determine the amount of tagged signal and normalization events by a two-dimensional extended maximum-likelihood fit to the neural network classifier output $\mathscr{O}_{N B}$ and $E_{\mathrm{ECL}}$. The latter variable is the sum of the energies of neutral clusters detected in the ECL that are not associated with reconstructed particles. Both signal and normalization events peak near zero in $E_{\mathrm{ECL}}$, while background events can populate a wider range. The likelihood function consists of the following five fit components: signal, normalization, events containing a wrongly reconstructed $D^{(*)}$ meson, $B \rightarrow D^{* *} \ell v_{\ell}$ events, and other backgrounds (predominantly from $B \rightarrow X_{c} D^{*}$ ). Three parameters are floated in the fit - the yields of the signal, normalization, and $B \rightarrow D^{* *} \ell v_{\ell}$ components. The result is shown in Fig. 1.

The yields of signal and normalization events are measured to be $231 \pm 23$ (stat) and $2800 \pm$ 57(stat), respectively. This can be converted into a result for $\mathscr{R}\left(D^{*}\right)$ by using the formula,

$$
\mathscr{R}\left(D^{*}\right)=\frac{1}{2 \mathscr{B}\left(\tau^{-} \rightarrow \ell^{-} \bar{v}_{\ell} \nu_{\tau}\right)} \frac{\varepsilon_{\text {norm }}}{\varepsilon_{\text {sig }}} \frac{N_{\text {sig }}}{N_{\text {norm }}},
$$

where $\varepsilon_{\mathrm{sig}(\text { norm })}$ and $N_{\mathrm{sig}(\text { norm) }}$ are the reconstruction efficiency and the yield of signal (normalization) events. We use $\mathscr{B}\left(\tau^{-} \rightarrow \ell^{-} \bar{v}_{\ell} v_{\tau}\right)=0.176 \pm 0.003$ as the mean value of the world averages 

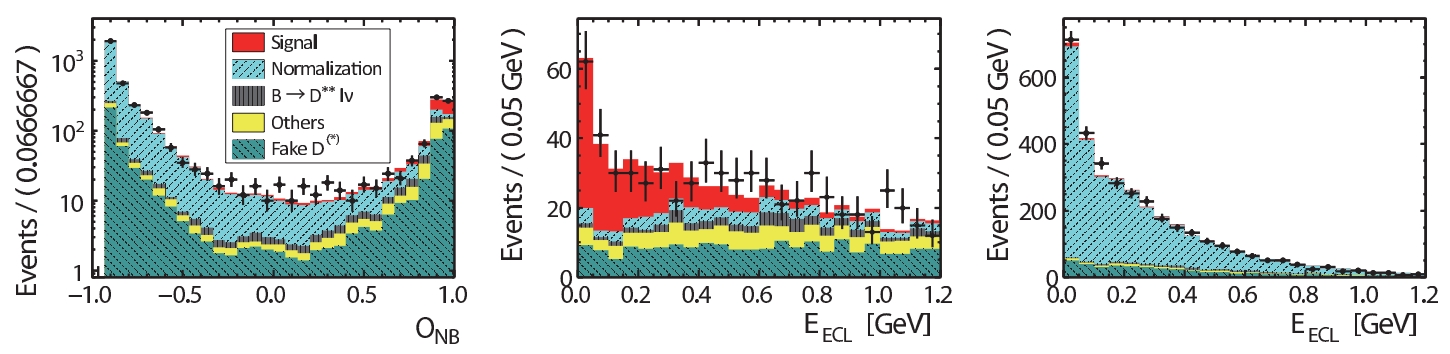

Figure 1: Measurement of the decay $B \rightarrow D^{*} \tau \nu$ with semileptonic tag: Projections of the fit results with data points overlaid for (left) the neural network classifier output, $\mathscr{O}_{N B}$, and the $E_{\mathrm{ECL}}$ distribution in (center) the signal-enhanced region, $\mathscr{O}_{N B}>0.8$, and (right) the normalization-enhanced region, $\mathscr{O}_{N B}<0.8$.

for $\ell=e$ and $\ell=\mu$ [13]. This results in

$$
\mathscr{R}\left(D^{*}\right)=0.302 \pm 0.030 \pm 0.011
$$

where the first uncertainty is statistical and the second systematic. The leading contribution to the systematic uncertainty results from the uncertainties in the PDF shapes used in the fit. The PDF uncertainty stems on the one hand from the limited size of the Monte Carlo simulated data sample but also from the limited knowledge of, e.g., the $B \rightarrow D^{* *} \ell v$ process.

This measurement is compatible with the determinations of $B \rightarrow D^{*} \tau \nu$ obtained by other experiments and brings the combined discrepancy of measurements of $B \rightarrow D^{(*)} \tau \nu$ with the SM expectation to the level of 4.0 standard deviations [14], Fig. 2.

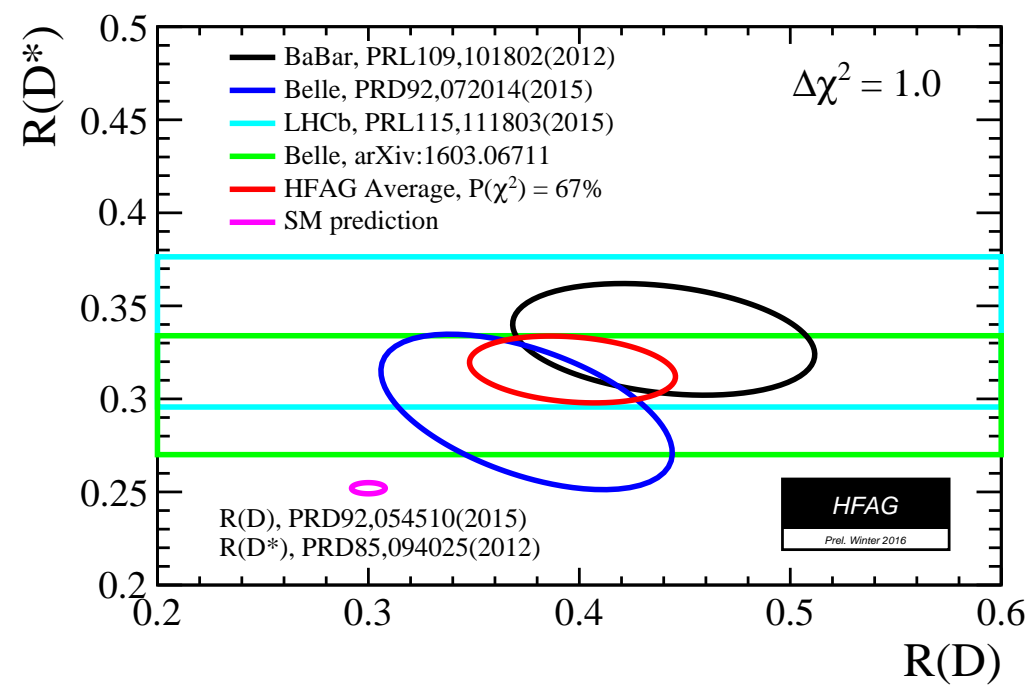

Figure 2: Combination of the new Belle measurement with previous measurements of $B \rightarrow D^{(*)} \tau \nu[5,6,7]$ by the Heavy Flavor Averaging Group [14]. The difference with the SM predictions is at $4.0 \sigma$ level.

\section{4. $B \rightarrow \pi \tau \nu$ with hadronic tagging}

To cross-check the excess seen in the $B \rightarrow D^{(*)} \tau \nu$ system, it is useful to search for the decay 
$B \rightarrow \pi \tau \nu$, where similar modifications would be expected in the presence of an extended Higgs sector. This is the motivation for the search for $B^{0} \rightarrow \pi^{-} \tau^{+} v$ in the full Belle data sample performed in Ref. [9].

This analysis uses the Belle full-reconstruction algorithm based on the NeuroBayes artificial neural network package [15]. There, 1104 hadronic decay topologies are searched for fully reconstructing one $B$ meson $\left(B^{0}\right.$ or $\left.B^{+}\right)$in a Belle $\Upsilon(4 S) \rightarrow B \bar{B}$ event (tag-side $B$ meson $B_{\text {tag }}$ ). To select good $B^{0}$ tags, we require the tag-side beam energy constrained mass,

$$
M_{\mathrm{bc}}=\sqrt{E_{\mathrm{beam}}^{2}-\left(\vec{p}_{B_{\mathrm{tag}}} c\right)^{2}} / c^{2}
$$

to be greater than $5.27 \mathrm{GeV} / \mathrm{c}^{2}$, where all quantities in the equation above are evaluated in the c.m. frame. In addition, the Neurobayes output $o_{\text {tag }}^{\mathrm{cs}}$, which quantifies the tag quality by a number between zero and 1 , is required to be greater than 0.18 .

On the signal-side, the decay $B^{0} \rightarrow \pi^{-} \tau^{+} v$ is searched for by reconstructing one-prong $\tau$ lepton decays into $e v v, \pi v$ and $\rho v$ (about $54 \%$ of the $\tau$ decay width). For signal events, we thus expect exactly two oppositely charged particles on the signal-side and significant missing mass $M_{\text {miss }}^{2}$ (as defined in the previous section) from the missing neutrino(s). Events which meet these requirements are selected. If the event contains two charged pions and a neutral pion candidate, we search for $\rho^{ \pm}$candidates, which are selected in a $\pi \pi^{0}$ mass range between 625 and $925 \mathrm{MeV} / \mathrm{c}^{2}$. Since $K_{L}$ mesons are not completely stopped in Belle, charmed $B$ decays with subsequent decays $D \rightarrow K_{L} \pi$ or $D \rightarrow K_{L} \ell v_{\ell}$ can mimic signal. Events with a KLM cluster without associated energy deposit in the ECL are vetoed.

To further improve background suppression, for each $\tau$ mode one boosted decision tree (BDT) is trained using the TMVA framework [16]. All BDTs use different input variables, background training samples, and BDT growth parameters. E.g.,, the input variables of the BDT used in the $\tau^{-} \rightarrow e^{-} \bar{v}_{e} \nu_{\tau}$ selection are the magnitude of the three-momenta of the pion and electron, the squared lepton-pair momentum transfer $q^{2}, M_{\text {miss }}^{2}$, and different combinations of all available fourmomenta.

Finally, to select signal candidates, we apply selections on the NeuroBayes output $o_{\text {tag }}^{\mathrm{cs}}$, the missing mass squared $M_{\text {miss }}^{2}$ and the BDT output. We perform a scan over these three variables simultaneously to obtain the optimal signal region for each $\tau$ mode. The signal yield is extracted by a binned maximum likelihood fit to the $E_{\mathrm{ECL}}$ distribution (as defined in the previous section), Fig. 3. There, signal is expected to accumulate near zero. The fit is performed simultaneously in all three reconstruction modes, with the signal strength parameter $\mu$ constrained between the three modes. Only the dominant $b \rightarrow c$ background is floated in the fit while the other, smaller components are fixed. The signal strength is chosen such that $\mu=1.0$ corresponds to $\mathscr{B}\left(B^{0} \rightarrow\right.$ $\left.\pi^{-} \tau^{+} v\right)=1.0 \times 10^{-4}$. We obtain a best fit of $\mu=1.52 \pm 0.72$, corresponding to $51.9 \pm 24.3$ signal events. The statistical significance of the signal is 2.8 standard deviations. Including systematic effects mainly from the uncertainty in tag-side reconstruction and the $K_{L}$ veto simulation, this is reduced to $2.4 \sigma$. At the $90 \%$ confidence level, we set an upper limit on the $B^{0} \rightarrow \pi^{-} \tau^{+} v$ branching fraction at $2.5 \times 10^{-4}$. 

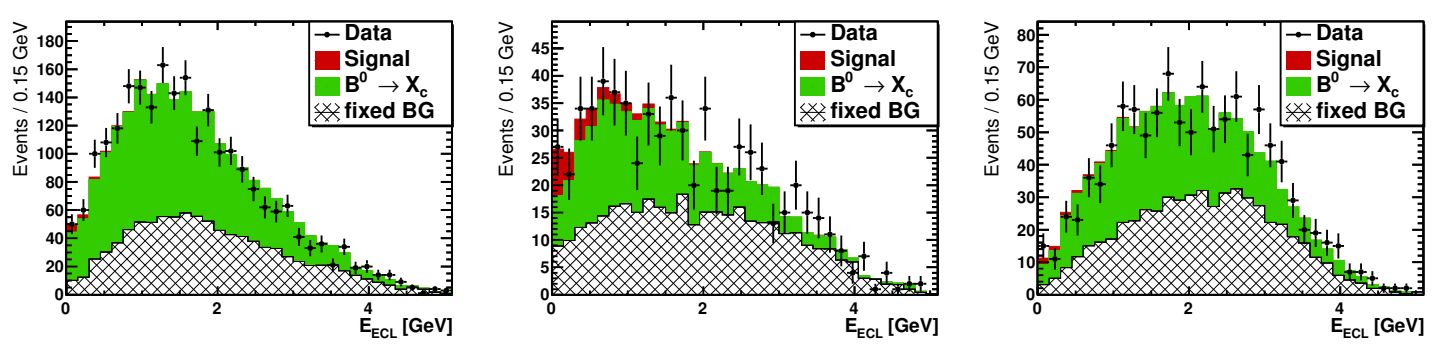

Figure 3: Search for $B \rightarrow \pi \tau v$ in fully-reconstructed Belle events: Fitted $E_{\mathrm{ECL}}$ distributions for the $\tau \rightarrow e v v$ (left), $\pi v$ (center) and $\rho v$ modes (right).

\section{Summary}

We have reviewed the two recent Belle measurements which examine semileptonic $B$ meson decays involving a $\tau$ lepton, a measurement of the decay $B \rightarrow D^{*} \tau \nu$ using the semileptonic tagging tag method [8], and a search for the decay $B \rightarrow \pi \tau \nu$ [9]. The former measurement confirms the excess seen in this mode by previous analyses and measures $\mathscr{R}\left(D^{*}\right)=0.302 \pm 0.030$ (stat) \pm 0.011 (syst) (see Eq. 3.1 for the definition of $\mathscr{R}$ ). The latter analysis fails to find a significant signal and sets a $90 \%$ confidence upper limit for $\mathscr{B}\left(B^{0} \rightarrow \pi^{-} \tau^{+} v\right)$ at $2.5 \times 10^{-4}$, which is compatible with the Standard Model expectation for this decay.

Both measurements are limited by the size of the Belle data set. Improvements of these studies can thus be expected from the next generation Belle II experiment, which will accumulate data starting from the year 2018.

\section{References}

[1] M. Tanaka, Z. Phys. C 67 (1995) 321 doi:10.1007/BF01571294 [hep-ph/9411405].

[2] H. Itoh, S. Komine and Y. Okada, Prog. Theor. Phys. 114 (2005) 179 doi:10.1143/PTP.114.179 [hep-ph/0409228].

[3] U. Nierste, S. Trine and S. Westhoff, Phys. Rev. D 78 (2008) 015006 doi:10.1103/PhysRevD.78.015006 [arXiv:0801.4938 [hep-ph]].

[4] S. Fajfer, J. F. Kamenik and I. Nisandzic, Phys. Rev. D 85 (2012) 094025 doi:10.1103/PhysRevD.85.094025 [arXiv:1203.2654 [hep-ph]].

[5] J. P. Lees et al. [BaBar Collaboration], Phys. Rev. Lett. 109 (2012) 101802 doi:10.1103/PhysRevLett.109.101802 [arXiv:1205.5442 [hep-ex]].

[6] M. Huschle et al. [Belle Collaboration], Phys. Rev. D 92 (2015) no.7, 072014 doi:10.1103/PhysRevD.92.072014 [arXiv:1507.03233 [hep-ex]].

[7] R. Aaij et al. [LHCb Collaboration], Phys. Rev. Lett. 115 (2015) no.11, 111803 Addendum: [Phys. Rev. Lett. 115 (2015) no.15, 159901] doi:10.1103/PhysRevLett.115.159901, 10.1103/PhysRevLett.115.111803 [arXiv:1506.08614 [hep-ex]].

[8] Y. Sato et al. [Belle Collaboration], arXiv:1607.07923 [hep-ex].

[9] P. Hamer et al. [Belle Collaboration], Phys. Rev. D 93 (2016) no.3, 032007 doi:10.1103/PhysRevD.93.032007 [arXiv:1509.06521 [hep-ex]]. 
[10] A. Abashian et al., Nucl. Instrum. Meth. A 479, 117 (2002).

[11] Charge conjugate decays are implied unless otherwise stated.

[12] M. Feindt and U. Kerzel, Nucl. Instrum. Methods Phys. Res., Sect. A 559, 190 (2006).

[13] K.A. Olive et al (Particle Data Group), Chin. Phys. C 38, 090001 (2014).

[14] http://www.slac.stanford.edu/xorg/hfag/semi/winter16/winter16_dtaunu.html

[15] M. Feindt, F. Keller, M. Kreps, T. Kuhr, S. Neubauer, D. Zander and A. Zupanc, Nucl. Instrum. Meth. A 654 (2011) 432 doi:10.1016/j.nima.2011.06.008 [arXiv:1102.3876 [hep-ex]].

[16] A. Hocker, P. Speckmayer, J. Stelzer, F. Tegenfeldt and H. Voss, CERN-2008-001. 